\title{
Evaluation of Prognostic Factors and Validation of Tumor Response Ratios after Neoadjuvant Chemotherapy in Patients with Breast
} Cancer

\author{
Tong Moon, Dong Hui Cho, Jung Min Youn ${ }^{1}$, Jae Bok Lee ${ }^{1}$, Jeoung Won Bae ${ }^{1}$, Seung Pil Jung ${ }^{1}$ \\ Division of Breast and Endocrine Surgery, Department of Surgery, Seoul Medical Center, Seoul; 'Division of Breast and Endocrine Surgery, \\ Department of Surgery, Korea University Hospital, Korea University College of Medicine, Seoul, Korea
}

Purpose: In the treatment of breast cancer, neoadjuvant chemotherapy (NAC) is useful to reduce breast cancer size before surgical intervention. Patients who achieve a pathologic complete response (pCR) to NAC have improved overall survival (OS). However, the relationship between prognosis and partial response is yet unclear. In this study, we evaluated prognostic factors and the tumor response ratio (TRR) method among patients who received NAC. Methods: Clinicopathologic factors were evaluated to predict OS. The TRR was calculated by dividing pathologic tumor size by clinical tumor size. TRRs were then categorized into four groups, and the survival times for the different TRR groups were compared using statistical evaluation. Results: Clinical $N$ stage $(p=0.02)$, overall stage $(p=0.04)$, pathologic $N$ stage $(p=0.03)$, hormone receptor status $(p=0.01)$, and lymphovascular invasion $(p=0.02)$ were significantly associated with OS. Pathologic overall stage and TRR did not correlate with OS. Patients with a PCR exhibited the best survival rates using the current staging system and the TRR method. Conclusion: Clinicopathologic factors can be easily applied to predict OS, and clinicians could use these parameters until an accurate, simple, and highly discriminatory methods is developed to assess breast cancer patients with a partial.

Key Words: Breast neoplasms, Neoadjuvant therapy, Prognosis, Response

\section{INTRODUCTION}

Cancer staging systems provide information that allows both clinicians and patients to design an optimal treatment and follow-up plan. Staging also allows for ease of communication among clinicians across multiple disciplines. Neoadjuvant chemotherapy (NAC) is the standard treatment for locally advanced breast cancer and has been increasingly implemented for treatment of patients with earlier stage operable cancer $[1,2]$. Because NAC results in improvements in disease-free survival (DFS) and overall survival (OS), which are similar to those obtained with adjuvant chemotherapy [3,4], NAC is used not only to reduce breast cancer size before surgical intervention but also to convert locally advanced inoperable breast cancer to operable disease [5-7].

Patients who show pathologic complete responses (pCRs) to NAC

\section{Correspondence: Seung Pil Jung}

Division of Breast and Endocrine Surgery, Department of Surgery, Korea University Hospital, Korea University College of Medicine, 73 Inchon-ro, Seongbuk-gu, Seoul 02841, Korea

Tel: +82-2-920-5978, Fax: +82-2-928-1631, E-mail: jungspil@korea.ac.kr Received: Sep 21, 2016 Revised: Oct 16, 2016 Accepted: Nov 21, 2016 have improved OS $[8,9]$. However, achieving a pCR is relatively uncommon, and there are only a few known predictive factors for OS in patients with partial responses [10-12]. Currently, breast cancer pathologic stage is evaluated using the American Joint Committee on Cancer (AJCC) tumor-node-metastasis classification system [13]. However, AJCC staging does not consider the effects of NAC, which is a common treatment modality for breast cancer. Clinical response to NAC would be a more precise way of describing the effect of NAC, and clinical response is evaluated according to the response evaluation criteria in solid tumors (RECIST) version 1.1 [14]. A pCR is defined as the complete disappearance of invasive cancer in both breast and axillary lymph nodes. Partial response is defined as a $>30 \%$ reduction in the largest tumor diameter. Progressive disease (PD) is defined as an increase of at least $20 \%$ from the base line diameter or the appearance of new disease. The lack of sufficient tumor shrinkage to qualify as partial response or a sufficient increase in tumor to qualify as PD is regarded as stable disease.

Several methods that attempt to predict the prognosis of patients with partial responses to NAC have been proposed [10,11,15]. However, these methods have not been properly validated and are limited be- 
cause of their complex calculations and unusual parameters. Therefore, no standard classification system for reporting responses to NAC exists. A method to predict survival rates of patients with partial responses to NAC would allow individualized treatment and surveillance. Therefore, we evaluated prognostic factors in a neoadjuvant setting. We also validated the tumor response ratio (TRR) method, which is a staging method for predicting the prognosis of breast cancer with a partial response after NAC, because this method is easily applicable using only clinical and pathologic tumor sizes and it accurately predicts OS [15].

\section{METHODS}

\section{Patients}

This study was performed retrospectively by using the records of 218 patients who received NAC to treat breast cancer between March 2006 and December 2013 at Seoul Medical Center and Korea University Hospital. Women who were treated with NAC and did not have distant metastasis at initial diagnosis were included in this study. Patient demographics and clinical characteristics were collected from the hospital's electronic database. Written informed consent was obtained from the patient or the patient's family. The study was approved by the Hospital Institutional Review Board (number: 2016105).

\section{Research methods}

Tumor location, operation method, histologic type, clinical and pathologic stage, hormone receptor status, human epidermal growth factor receptor 2 (HER2) status, tumor grade, presence of lymphovascular invasion (LVI), and margin status were evaluated as prognostic factors for survival after NAC.

The TRR method was implemented as previously described [15]. Tumor size prior to NAC was determined at the time of diagnosis using imaging modalities. Most patients were diagnosed using multiple imaging techniques. Magnetic resonance imaging (MRI) was the method of choice in this study; however, if MRI results were not available, ultrasonography was used as an alternative method. Only the diameter of the largest mass was used as a factor for TRR. Pathologic tumor sizes were obtained from pathologic reports after breast resection surgery. TRR was defined as (pathologic tumor size/prechemotherapy tumor size), and values were categorized into four risk groups, as in a previously published TRR study [15]. TRR 0 (pCR) signified complete remission, TRR 0 to 0.4 signified a strong partial response (SPR), TRR 0.4 to 1.0 signified a weak partial response (WPR), and TRR $>1.0$ signified tumor growth (TG). Ductal carcinoma in situ was not considered.

OS was measured as the time from breast cancer diagnosis until death from any cause or until the last follow-up date. Estrogen and progesterone receptor statuses were evaluated in the primary tumor using standard immunohistochemistry (Dako, Glostrup, Denmark); tumors were considered positive if more than $1 \%$ of cells exhibited nuclear staining. HER2 status was determined in primary tumor tissues using the anti-HER2 monoclonal antibody (Lab Vision Corp., Fremont, USA), and staining was considered positive if a staining intensity score of 2 along with fluorescence in situ hybridization positivity or a score of 3 alone were obtained.

\section{Statistical analysis}

To evaluate patient characteristics, the frequency analysis method was used. The Kaplan-Meier method and log-rank test were used to estimate survival, according to clinicopathologic factors. Using Cox hazard regression analysis, multivariate analyses were conducted to evaluate the effects of independent variables on dependent variables. Statistical analyses were performed using PASW Statistics version 18.0 (SPSS Inc., Chicago, USA). Reported $p$-values are two-sided, and statistical significance was set at $p<0.05$.

\section{RESULTS}

\section{Patients}

Table 1 shows the patient clinicopathologic characteristics. The mean age of our study group was 49 years at the time of diagnosis. Of 218 patients, $179(82.1 \%)$ were assessed for pretreatment cancer size using MRI. Thirty-nine patients (17.9\%) did not undergo MRI; therefore, ultrasonography was used for assessing tumor size. Most patients were diagnosed with invasive ductal carcinoma $(n=191,87.6 \%)$, clinical T2 lesions ( $\mathrm{n}=139,63.8 \%$ ), and clinical N1 tumors $(\mathrm{n}=96,44.0 \%)$. For pathologic stage, ypT1 $(n=86,39.5 \%)$ and ypN0 $(n=97,44.5 \%)$ were the most common, and for final pathologic stage, stage II $(n=78$, $35.8 \%$ ) was the most common. The dominant disease subtypes were 
Table 1. Patient clinicopathologic characteristics $(n=218)$

\begin{tabular}{|c|c|c|c|c|c|}
\hline Characteristic & No. (\%) & Mortality & Characteristic & No. (\%) & Mortality \\
\hline Age at diagnosis (yr)* & $48.75 \pm 10.0$ & - & Clinical overall stage & & \\
\hline Location & & & I & $3(1.4)$ & 0 \\
\hline Right & $114(52.3)$ & 13 & $\|$ & $101(46.3)$ & 7 \\
\hline Left & $102(46.8)$ & 10 & III & $114(52.3)$ & 16 \\
\hline Both & $2(0.9)$ & 0 & Pathologic overall stage & & \\
\hline Method of tumor assessment & & & 0 & $36(16.5)$ & 1 \\
\hline MRI & $179(82.1)$ & - & I & $42(19.3)$ & 3 \\
\hline Ultrasonography & $39(17.9)$ & - & $\|$ & $78(35.8)$ & 8 \\
\hline Operation method & & & III & $62(28.4)$ & 11 \\
\hline Conserving surgery & $122(56.0)$ & 7 & Tumor response ratio & & \\
\hline Mastectomy & $96(44.0)$ & 16 & $0 \mathrm{pCR}$ & $41(18.8)$ & 2 \\
\hline Histology & & & $0.0-0.4$ SPR & $66(30.3)$ & 8 \\
\hline Invasive ductal & $191(87.6)$ & 19 & $0.4-1.0 \mathrm{WPR}$ & 87 (39.9) & 9 \\
\hline Invasive ductal mixed & $5(2.3)$ & 0 & $>1.0 \mathrm{TG}$ & $24(11.0)$ & 4 \\
\hline Invasive lobular & $4(1.8)$ & 0 & Hormone receptor status & & \\
\hline Others & $18(8.3)$ & 4 & Negative & $118(54.1)$ & 16 \\
\hline Clinical T stage & & & Positive & $98(45.0)$ & 7 \\
\hline $\mathrm{T} 1$ & $26(11.9)$ & 1 & Unknown & $2(0.9)$ & 0 \\
\hline $\mathrm{T} 2$ & $139(63.8)$ & 13 & HER2 receptor status & & \\
\hline $\mathrm{T} 3$ & $31(14.2)$ & 3 & Negative & $144(66.1)$ & 13 \\
\hline $\mathrm{T} 4$ & $22(10.1)$ & 6 & Positive & $72(33.0)$ & 10 \\
\hline Pathologic ypT stage & & & Unknown & $2(0.9)$ & \\
\hline TO & $40(18.3)$ & 1 & Histologic grade & & \\
\hline $\mathrm{T} 1$ & $86(39.5)$ & 8 & Low & $14(6.4)$ & 1 \\
\hline $\mathrm{T} 2$ & $69(31.7)$ & 6 & Intermediate & $69(31.7)$ & 4 \\
\hline $\mathrm{T} 3$ & $16(7.3)$ & 6 & High & $77(35.3)$ & 15 \\
\hline $\mathrm{T} 4$ & $7(3.2)$ & 2 & Unknown & $58(26.6)$ & 3 \\
\hline Clinical N stage & & & Lymphovascular invasion & & \\
\hline No & $32(14.7)$ & 2 & No & $153(70.2)$ & 9 \\
\hline $\mathrm{N} 1$ & $96(44.0)$ & 6 & Yes & $65(29.8)$ & 23 \\
\hline N2 & $54(24.8)$ & 7 & Resection margin & & \\
\hline N3 & $36(16.5)$ & 8 & Not involved & $213(97.7)$ & 23 \\
\hline Pathologic ypN stage & & & Involved & $5(2.3)$ & 0 \\
\hline NO & $97(44.5)$ & 6 & & & \\
\hline $\mathrm{N} 1$ & $73(33.5)$ & 7 & & & \\
\hline N2 & $33(15.1)$ & 6 & & & \\
\hline N3 & $15(6.9)$ & 4 & & & \\
\hline
\end{tabular}

$\mathrm{MRI}=$ magnetic resonance imaging; $\mathrm{PCR}=$ pathologic complete response; $\mathrm{SPR}=$ strong partial response; $\mathrm{WPR}=$ weak partial response; $\mathrm{TG}=$ tumor growth; HER2 = human epidermal growth factor receptor 2.

*Mean \pm SD.

Table 2. Tumor response rate and the incidence of any cause mortality according to receptor status

\begin{tabular}{lcccccc}
\hline & Total & PCR, No. (\%) & SPR, No. (\%) & WPR, No. (\%) & TG, No. (\%) & Mortality, No. (\%) \\
\hline $\begin{array}{l}\text { Overall cohort } \\
\text { Receptor status }\end{array}$ & 218 & $41(18.8)$ & $66(30.3)$ & $87(39.9)$ & $24(11.0)$ & $23(10.6)$ \\
$\quad$ HR (+), HER2 (-) & & & & & & \\
HR (+), HER2 (+) & 71 & $10(13.2)$ & $24(31.6)$ & $29(38.2)$ & $8(10.5)$ & $5(7.0)$ \\
HR (-), HER2 (+) & 27 & $3(10.3)$ & $7(24.1)$ & $15(51.7)$ & $2(6.9)$ & $2(7.4)$ \\
HR (-), HER2 (-) & 45 & $11(24.4)$ & $13(28.9)$ & $16(35.6)$ & $5(11.1)$ & $8(17.7)$ \\
HR (unknown), HER2 (unknown) & 73 & $17(25.8)$ & $21(31.8)$ & $27(40.9)$ & $8(12.1)$ & $8(10.9)$ \\
\end{tabular}

$\mathrm{pCR}=$ pathologic complete response; $\mathrm{SPR}=$ strong partial response; $\mathrm{WPR}=$ weak partial response; $\mathrm{TG}=$ tumor growth; $\mathrm{HR}=$ hormone receptor; $\mathrm{HER} 2=$ human epidermal growth factor receptor 2 . 
Table 3. The prognostic factor for overall survival using univariate and multivariate analyses

\begin{tabular}{|c|c|c|c|c|}
\hline Characteristic & Unadjusted 5-yr survival $(95 \% \mathrm{Cl})$ & $p$-value (uni)* & Adjusted hazard ratio $(95 \% \mathrm{Cl})$ & $p$-value $\left(\right.$ multi) ${ }^{\dagger}$ \\
\hline Operation method & & 0.01 & & 0.26 \\
\hline Conserving surgery & $0.91(0.87-0.95)$ & & 1 & \\
\hline Mastectomy & $0.80(0.71-0.81)$ & & $1.87(0.89-2.32)$ & \\
\hline Clinical T stage & & 0.04 & & 0.34 \\
\hline $\mathrm{T} 1$ & $0.94(0.93-0.95)$ & & 1 & \\
\hline $\mathrm{T} 2$ & $0.90(0.87-0.93)$ & & $1.34(0.37-3.24)$ & \\
\hline T3 & $0.85(0.80-0.90)$ & & $1.75(0.53-3.98)$ & \\
\hline T4 & $0.62(0.45-0.74)$ & & $2.56(0.78-5.32)$ & \\
\hline Pathologic ypT stage & & $<0.01$ & & 0.77 \\
\hline T0 & $0.97(0.93-0.99)$ & & 1 & \\
\hline $\mathrm{T} 1$ & $0.86(0.83-0.91)$ & & $1.64(0.48-2.91)$ & \\
\hline $\mathrm{T} 2$ & $0.81(0.74-0.89)$ & & $1.82(0.78-5.62)$ & \\
\hline $\mathrm{T} 3$ & $0.53(0.37-0.67)$ & & $3.75(0.81-11.23)$ & \\
\hline $\mathrm{T} 4$ & $0.43(0.22-0.64)$ & & $4.26(0.83-17.16)$ & \\
\hline Clinical N stage & & 0.02 & & 0.02 \\
\hline No & $0.89(0.82-0.96)$ & & 1 & \\
\hline N1 & $0.81(0.77-0.82)$ & & $1.63(1.17-2.34)$ & \\
\hline N2 & $0.76(0.67-0.83)$ & & $1.89(1.34-3.12)$ & \\
\hline N3 & $0.51(0.35-0.69)$ & & $2.78(1.12-5.45)$ & \\
\hline Pathologic ypN stage & & $<0.01$ & & 0.03 \\
\hline No & $0.89(0.86-0.92)$ & & 1 & \\
\hline N1 & $0.83(0.78-0.88)$ & & $1.58(1.02-3.15)$ & \\
\hline N2 & $0.68(0.57-0.79)$ & & $2.38(1.93-6.01)$ & \\
\hline N3 & $0.36(0.14-0.58)$ & & $3.25(1.45-8.65)$ & \\
\hline Clinical overall stage & & 0.02 & & 0.04 \\
\hline I & 1.00 & & 1 & \\
\hline$\|$ & $0.89(0.86-0.92)$ & & $1.65(1.26-4.17)$ & \\
\hline III & $0.82(0.77-0.86)$ & & $2.15(1.45-6.56)$ & \\
\hline Pathologic overall stage & & 0.04 & & 0.07 \\
\hline 0 & $0.96(0.94-0.98)$ & & 1 & \\
\hline I & $0.87(0.82-0.92)$ & & $1.46(0.81-2.31)$ & \\
\hline$\|$ & $0.83(0.78-0.88)$ & & $2.15(0.97-6.32)$ & \\
\hline III & $0.69(0.61-0.77)$ & & $2.98(1.45-8.32)$ & \\
\hline Tumor response ratio & & 0.03 & & 0.06 \\
\hline $0 \mathrm{pCR}$ & $0.92(0.88-0.94)$ & & 1 & \\
\hline $0.0-0.4$ SPR & $0.82(0.78-0.86)$ & & $1.73(0.93-4.65)$ & \\
\hline 0.4-1.0WPR & $0.80(0.75-0.85)$ & & $2.56(1.00-8.34)$ & \\
\hline$>1.0 \mathrm{TG}$ & $0.71(0.59-0.83)$ & & $3.14(2.15-13.45)$ & \\
\hline Hormone receptor status & & $<0.01$ & & 0.01 \\
\hline Negative & $0.81(0.76-0.86)$ & & 1 & \\
\hline Positive & $0.87(0.82-0.92)$ & & $0.75(0.23-0.87)$ & \\
\hline Unknown & $\mathrm{N} / \mathrm{A}$ & & $\mathrm{N} / \mathrm{A}$ & \\
\hline Histologic grade & & $<0.01$ & & 0.13 \\
\hline Low & $0.85(0.72-0.98)$ & & 1 & \\
\hline Intermediate & $0.90(0.85-0.95)$ & & $1.47(0.45-3.56)$ & \\
\hline High & $0.69(0.62-0.75)$ & & $3.14(0.78-7.75)$ & \\
\hline Unknown & $0.89(0.86-0.92)$ & & $1.98(0.63-5.59)$ & \\
\hline Lymphovascular invasion & & $<0.01$ & & 0.02 \\
\hline No & $0.93(0.90-0.96)$ & & 1 & \\
\hline Yes & $0.61(0.52-0.70)$ & & $1.68(1.13-3.12)$ & \\
\hline
\end{tabular}

$\mathrm{Cl}=$ confidence interval; $\mathrm{pCR}=$ pathologic complete response; $\mathrm{SPR}=$ strong partial response; $\mathrm{WPR}=$ weak partial response; $\mathrm{TG}=$ tumor growth; $\mathrm{N} / \mathrm{A}=$ not available.

${ }^{*}$ Overall unadjusted survival tested with the log-rank test; ${ }^{\dagger}$ Overall adjusted survival tested with Cox regression. 
hormone receptor-negative $(\mathrm{n}=118,54.1 \%)$ and HER2-negative $(\mathrm{n}=144,66.1 \%)$. Most patients were negative for $\mathrm{LVI}(\mathrm{n}=153,70.2 \%)$.

\section{Tumor response ratio}

There were 41 patients (18.8\%) in the pCR category. Sixty-six patients (30.3\%) were in the SPR group, 87 patients (39.9\%) were in the WPR group, and 24 patients (11.0\%) were in the TG group. Table 2 shows the TRR according to receptor status and mortality. More than $20 \%$ of patients in the hormone receptor-negative group achieved a pCR, whereas, about $10 \%$ of the hormone receptor-positive group showed a pCR. Although the pCR rate was higher in the hormone receptor-negative group, mortality was more frequently observed in this group.

\section{Clinical outcomes}

During a mean follow-up of 44 months, 61 patients (28.0\%) experienced locoregional and/or distant disease recurrences, and 23 patients (10.6\%) died. Table 1 shows the number of patients who died of any cause, and Table 3 shows statistically significant factors based on univariate and multivariate analyses. In univariate analysis using the Kaplan-Meier method, mastectomy $(p=0.01)$, clinical T stage $(p=0.04)$, clinical N stage $(p=0.02)$, overall clinical stage $(p=0.02)$ (Figure 1 ), pathologic T stage $(p<0.01)$ and pathologic $\mathrm{N}$ stage $(p<0.01)$ predicted worse OS. Patients with breast cancer that tested positive for hormone receptor $(p<0.01)$, was of low histologic grade $(p<0.01)$, and

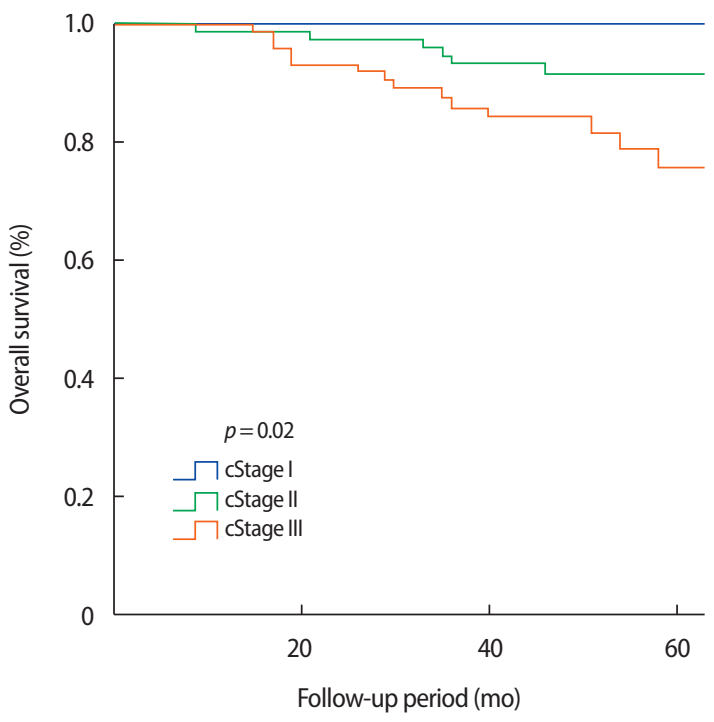

Figure 1. Kaplan-Meier analysis of overall survival according to clinical stage. was without LVI $(p<0.01)$ (Figure 2) experienced better OS. TRR was also a statistically significant predictor of OS ( $p=0.03$ ) (Figure 3 ), as lower TRR ratios were significantly associated with better OS using Kaplan-Meier univariate analysis.

Significant factors from univariate analyses were assessed by multivariate analyses using the Cox regression method to evaluate prognostic significance (Table 3$)$. Clinical $\mathrm{N}$ stage $(p=0.02)$, clinical overall

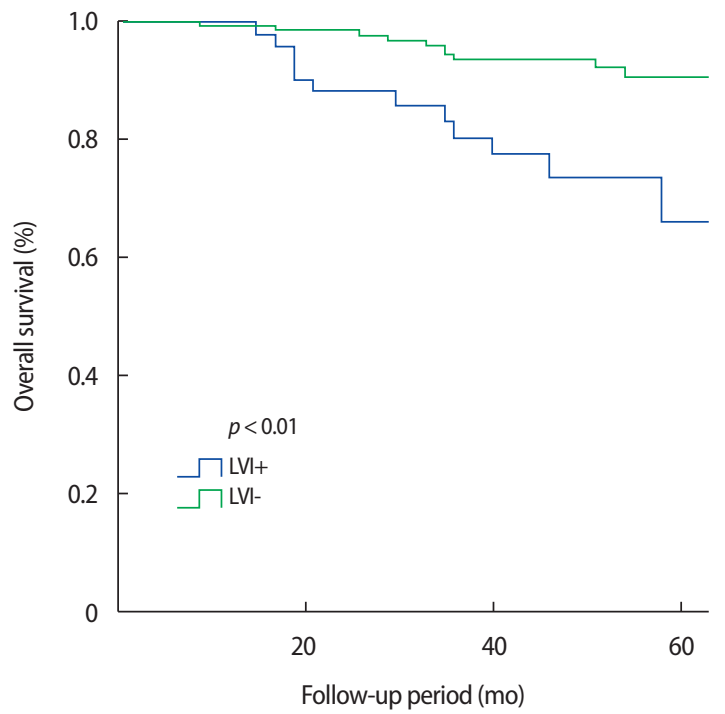

Figure 2. Kaplan-Meier analysis of overall survival according to lymphovascular invasion (LVI).

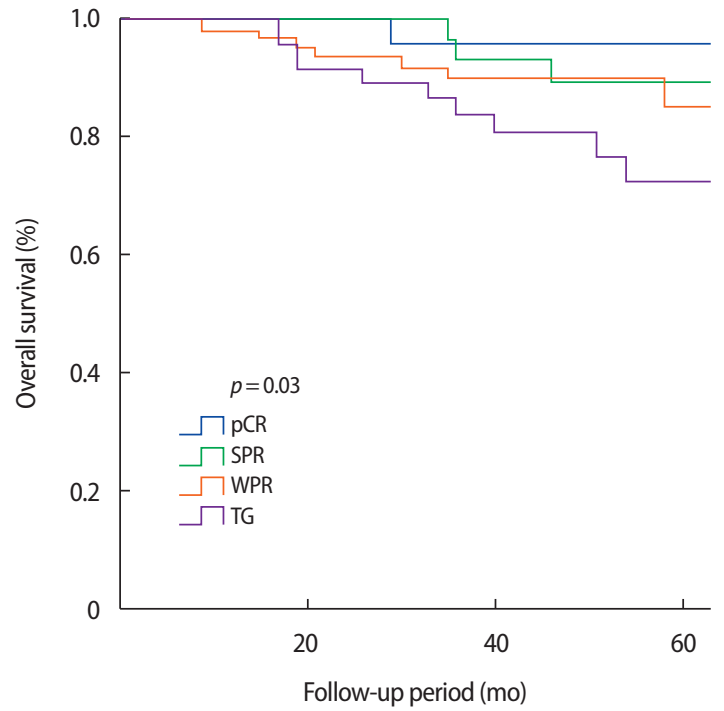

Figure 3. Kaplan-Meier analysis of overall survival according to tumor response ratio group.

$\mathrm{pCR}=$ pathologic complete response; $\mathrm{SPR}=$ strong partial response; WPR=weak partial response; $\mathrm{TG}=$ tumor growth. 
stage $(p=0.04)$, pathologic $\mathrm{N}$ stage $(p=0.03)$, hormone receptor status ( $p=0.01)$, and LVI $(p=0.02)$ were significantly associated with OS. Although pathologic overall stage and TRR tended to predict good responses and better OS, they were not statistically significant. However, patients with pathologic complete remission had the best survival rate when using the current pathologic staging and TRR methods.

\section{DISCUSSION}

The wide use of NAC has made breast cancer staging more complex. Unfortunately, the current staging system cannot incorporate the response to NAC. It is unclear whether initial clinical stage or postNAC pathologic stage is more significant for prognosis. Therefore, efforts have been made to examine the predictive value of clinicopathologic factors and tumor responses to NAC $[8,10,11,16]$. Pathologic complete remission is a well-known predictive factor for improved OS $[8,16]$. Additionally, the degree of tumor response and the extent of residual disease after NAC are associated with survival. However, there is no standard classification system for reporting responses to NAC. Therefore, we evaluated the prognostic value and utility of TRR methods to predict survival after NAC for breast cancer.

In this study, multivariate analysis showed that clinical $\mathrm{N}$ stage $(p=0.02)$, clinical overall stage $(p=0.04)$, pathologic $\mathrm{N}$ stage $(p=0.03)$, hormone receptor status $(p=0.01)$, and LVI $(p=0.02)$ were effective factors associated with OS. Although pathologic overall stage and TRR system values were not significantly associated with survival, they exhibited a trend toward association with OS

Several methods are used to predict survival of patients who receive NAC. Symmans et al. [10] used residual cancer burden to improve prognostication. This method significantly predicted relapse-free survival. The calculation involves the primary tumor bed diameter, the amount of residual primary invasive cancer, the number of positive lymph nodes, and the largest metastatic lymph node size. This method is difficult to use because required data may not be readily available after routine pathologic assessments. Sataloff et al. [17] proposed a scale from A to D to grade histological responses for both residual primary tumors and axillary disease. However, both of these systems rely heavily on subjective assessments by pathologists and show a lack of statistical significance when predicting OS. The TRR system introduced by Miller et al. [15] requires only clinical and pathologic tumor size accurately to predict OS. However, this has not been properly validated. Therefore, we selected this method because of its easy application, and the opportunity for proper validation. Although TRR implementation resulted in accurate prognosis in univariate analysis ( $p=0.03)$, TRR was not a statistically significant predictor by multivariate analysis $(p=0.06)$. This discordance between our study and that of by Miller et al. [15] could be because our study was conducted in patients with different characteristics. Our study included patients with a higher clinical $\mathrm{N}$ stage than the previous study. In our study, more than $20 \%$ of patients had clinical N2 and 3 stage disease, whereas, in the study by Miller et al. [15], only 3\% of patients had clinical N2 and 3 stage disease. As shown in the results, clinical $\mathrm{N}$ stage is significant prognostic factor for OS; this stage difference could be one of reason for the discordance. Second, our study was conducted in Korean patients, but the previous study was conducted in American patients, of whom only 13\% were of Asian origin. Asian patients have different characteristics from American patients. A higher proportion of young Asian women are diagnosed with breast cancer compared to their Caucasian and Hispanic counterparts. Furthermore, they are more likely to present with unfavorable tumor characteristics, such as lymph node involvement, large tumor size, HER2 expression, and estrogen receptor-negative disease [18,19]. This ethnic difference could affect the accuracy of the TRR method.

TRR method uses the largest diameter of pretreatment and pathologic tumor size. Some tumors shrink concentrically, while others are broken down to scattered islands. Therefore, assessing tumor volume may be more accurate than assessing the diameter. Unfortunately, there are some technical limitations to assessing accurate tumor volume. In our two hospitals, standard pathology provided only the largest tumor diameter without 3-dimensional tumor volume. This retrospective study could not assess postoperative tumor volume due to the storage method of surgical specimens, which were kept as divided blocks rather than in their intact shape.

Although TRR and pathologic stage were not statistically significant predictors, patients with a pCR had the best OS. The goal of chemotherapy administered in the adjuvant or neoadjuvant setting is to eradicate occult distant metastases to ultimately improve DFS. Theoretically, if a CR is reflective of the chemosensitivity of occult distant metastases, patients who achieve a pCR might have the highest DFS rates, compared with those with lesser responses. 
In this study, we showed that clinical $\mathrm{N}$ stage, clinical overall stage, pathologic $\mathrm{N}$ stage, hormone receptor status, and LVI are statistically significant prognostic factors for all patients after NAC. These factors are easily applicable because they require only imaging studies and pathology reports, which are obtained in normal practice. Until an accurate, simple, and highly discriminatory method is developed to assess breast cancer with a partial response, clinicopathologic factors are still valuable for predicting OS.

This study is limited by several factors. First, the data are from a small cohort of patients who were heterogeneously treated, as well as a high-risk population treated with aggressive multimodal therapy. Furthermore, different imaging techniques, including MRI and ultrasonography, were used to assess pretreatment tumor size. We chose MRI as the main method in this study because it is highly sensitive for detecting invasive breast cancer and shows excellent correlation between the gross histology size and the size determined using MRI [20,21]. Thirty-nine patients were assessed using ultrasonography, which may not be comparable. For this reason, our results may not be generalizable until multi-institution, large cohort studies with longer follow-up durations are performed.

In this study, we could not validate the efficacy of TRR for patients with a partial response. However, we found useful clinicopathologic prognostic factors for all patients and patients with a partial response after NAC. As these factors are easily applicable, clinicians can use them to predict the prognosis after NAC for all patients, including those with a partial response.

\section{CONFLICT OF INTEREST}

The authors declare that they have no competing interests.

\section{REFERENCES}

1. Bonadonna G, Valagussa P, Rossi A, Zucali R, Tancini G, Bajetta E, et al. Are surgical adjuvant trials altering the course of breast cancer? Semin Oncol 1978;5:450-64.

2. Kantarjian HM, Hortobagyi GN, Smith TL, Blumenschein GR, Montague E, Buzdar AU, et al. The management of locally advanced breast cancer: a combined modality approach. Eur J Cancer Clin Oncol 1984;20:1353-61.
3. Rastogi P, Anderson SJ, Bear HD, Geyer CE, Kahlenberg MS, Robidoux A, et al. Preoperative chemotherapy: updates of National Surgical Adjuvant Breast and Bowel Project Protocols B-18 and B-27. J Clin Oncol 2008;26:778-85.

4. Berruti A, Generali D, Kaufmann M, Puztai L, Curigliano G, Aglietta $\mathrm{M}$, et al. International expert consensus on primary systemic therapy in the management of early breast cancer: highlights of the fourth Symposium on Primary Systemic Therapy in the Management of Operable Breast Cancer, Cremona, Italy (2010). J Natl Cancer Inst Monogr 2011;2011:147-51.

5. Bonadonna G, Veronesi U, Brambilla C, Ferrari L, Luini A, Greco M, et al. Primary chemotherapy to avoid mastectomy in tumors with diameters of three centimeters or more. J Natl Cancer Inst 1990; 82:1539-45.

6. Fisher B, Brown A, Mamounas E, Wieand S, Robidoux A, Margolese RG, et al. Effect of preoperative chemotherapy on local-regional disease in women with operable breast cancer: findings from $\mathrm{Na}$ tional Surgical Adjuvant Breast and Bowel Project B-18. J Clin Oncol 1997;15:2483-93.

7. Fisher B, Bryant J, Wolmark N, Mamounas E, Brown A, Fisher ER, et al. Effect of preoperative chemotherapy on the outcome of women with operable breast cancer. J Clin Oncol 1998;16:2672-85.

8. Kuerer HM, Newman LA, Smith TL, Ames FC, Hunt KK, Dhingra $\mathrm{K}$, et al. Clinical course of breast cancer patients with complete pathologic primary tumor and axillary lymph node response to doxorubicin-based neoadjuvant chemotherapy. J Clin Oncol 1999; 17:460-9.

9. Lippman ME, Sorace RA, Bagley CS, Danforth DW Jr, Lichter A, Wesley MN. Treatment of locally advanced breast cancer using primary induction chemotherapy with hormonal synchronization followed by radiation therapy with or without debulking surgery. NCI Monogr 1986;(1):153-9.

10. Symmans WF, Peintinger F, Hatzis C, Rajan R, Kuerer H, Valero V, et al. Measurement of residual breast cancer burden to predict survival after neoadjuvant chemotherapy. J Clin Oncol 2007;25:441422.

11. Jeruss JS, Mittendorf EA, Tucker SL, Gonzalez-Angulo AM, Buchholz TA, Sahin AA, et al. Staging of breast cancer in the neoadjuvant setting. Cancer Res 2008;68:6477-81.

12. Kim J, Lee J, Chang E, Suh K, Lee C, Jee J, et al. Prognostic factors in 
patients with stage II/III breast cancer treated with adjuvant extension of neoadjuvant chemotherapy: a retrospective cohort study with ten-years of follow-up data. J Breast Cancer 2011;14:39-45.

13. Edge SB; American Joint Committee on Cancer. AJCC Cancer Staging Manual. 7th ed. New York: Springer; 2010.

14. Eisenhauer EA, Therasse P, Bogaerts J, Schwartz LH, Sargent D, Ford R, et al. New response evaluation criteria in solid tumours: revised RECIST guideline (version 1.1). Eur J Cancer 2009;45:228-47.

15. Miller M, Ottesen RA, Niland JC, Kruper L, Chen SL, Vito C. Tumor response ratio predicts overall survival in breast cancer patients treated with neoadjuvant chemotherapy. Ann Surg Oncol 2014;21: 3317-23.

16. Carey LA, Metzger R, Dees EC, Collichio F, Sartor CI, Ollila DW, et al. American Joint Committee on Cancer tumor-node-metastasis stage after neoadjuvant chemotherapy and breast cancer outcome. J Natl Cancer Inst 2005;97:1137-42.

17. Sataloff DM, Mason BA, Prestipino AJ, Seinige UL, Lieber CP, Ba- loch Z. Pathologic response to induction chemotherapy in locally advanced carcinoma of the breast: a determinant of outcome. J Am Coll Surg 1995;180:297-306.

18. Agarwal G, Pradeep PV, Aggarwal V, Yip CH, Cheung PS. Spectrum of breast cancer in Asian women. World J Surg 2007;31:103140.

19. Telli ML, Chang ET, Kurian AW, Keegan TH, McClure LA, Lichtensztajn D, et al. Asian ethnicity and breast cancer subtypes: a study from the California Cancer Registry. Breast Cancer Res Treat 2011; 127:471-8.

20. Weinreb JC, Newstead G. MR imaging of the breast. Radiology 1995;196:593-610.

21. Amano G, Ohuchi N, Ishibashi T, Ishida T, Amari M, Satomi S. Correlation of three-dimensional magnetic resonance imaging with precise histopathological map concerning carcinoma extension in the breast. Breast Cancer Res Treat 2000;60:43-55. 